\title{
Correction to: Effect of the rotation and tidal dissipation history of stars on the evolution of close-in planets
}

\author{
Emeline Bolmont ${ }^{1,2,3} \cdot$ Stéphane Mathis ${ }^{2}$
}

Published online: 24 January 2019

(c) Springer Nature B.V. 2019

\section{Correction to: Celest Mech Dyn Astr (2016) 126:275-296 https://doi.org/10.1007/s10569-016-9690-3}

\begin{abstract}
This is an erratum for the publication Bolmont and Mathis 2016 (Celest Mech Dyn Astr 126:275-296). There was a small mistake for the spin integration of our code which we corrected and we take advantage of this erratum to investigate a bit further the influence of a planet on the spin of its host star.
\end{abstract}

Keywords Planets and satellites: dynamical evolution and stability - Planet-star interactions - Planets and satellites: terrestrial planets - Planets and satellites: gaseous planets - stars: evolution - stars: rotation

The code used in Bolmont and Mathis (2016) had a small mistake for the spin integration. While this does not change the results of the simulations and most of the plots; the correction of this bug has an impact on Fig. 5 .

Figure 5 displayed the difference between the rotation period of a star with an outwardmigrating planet and the rotation of the same star without planet. This figure has the purpose of showing the impact of the planet on the rotation of its host star. The variations of the stellar rotation period induced by the migration of the planet are quite small compared to the spin-up and spin-down amplitude; plotting the difference allows to magnify and isolate this effect.

We take advantage of this erratum where we corrected our code to explain and investigate the evolution of the rotation period of a star with a migrating planet compared to the evolution of the rotation period of a star without planet. We define here $\delta P$ as the difference between the rotation period of the star with planet $P_{\star, \mathrm{pl}}$ and the rotation period of the star without planet $P_{\star, \text { no pl }}$

The original article can be found online at https://doi.org/10.1007/s10569-016-9690-3.

Emeline Bolmont

emeline.bolmont@unige.ch

1 NaXys, Department of Mathematics, University of Namur, 8 Rempart de la Vierge, 5000 Namur, Belgium

2 Laboratoire AIM Paris-Saclay, CEA/DRF - CNRS - Univ. Paris Diderot - IRFU/SAp, Centre de Saclay, 91191 Gif-sur-Yvette Cedex, France

3 Observatoire Astronomique de l'Université de Genève, Université de Genève, Chemin des Maillettes 51, 1290 Versoix, Switzerland 


$$
\delta P(t)=P_{\star, \mathrm{pl}}(t)-P_{\star, \text { no } \mathrm{pl}}(t) .
$$

Similarly we define $\delta \Omega=\Omega_{\star, \mathrm{pl}}(t)-\Omega_{\star, \mathrm{no} \mathrm{pl}}(t)$ as the difference between the spin of the star with planet and the spin of the star without planet. $\Omega_{\star}$ is here the angular velocity of the star: $\Omega_{\star}=2 \pi / P_{\star}$.

The star is considered here to have a solid rotation. The planets are on coplanar and circular orbits. We remind here the equations governing the evolution of the spin $\Omega_{\star}$ of the star (Eq. 14 of Bolmont and Mathis 2016)

$$
\begin{aligned}
\frac{\mathrm{d} I_{\star} \Omega_{\star}}{\mathrm{d} t}= & -K \Omega_{\star}^{\mu} \omega_{\text {sat }}^{3-\mu}\left(\frac{R_{\star}}{R_{\odot}}\right)^{1 / 2}\left(\frac{M_{\star}}{M_{\odot}}\right)^{-1 / 2} \\
& +\frac{h}{2 T_{\star}}\left[1-\frac{\Omega_{\star}}{n}\right]
\end{aligned}
$$

where $I_{\star}$ is the stellar moment of inertia which varies as the radius of the star $R_{\star}$ evolves (we use the evolution grids of Siess et al. 2000), $h$ the orbital angular momentum, $n$ the mean orbital angular frequency, $T_{\star}$ the stellar dissipation timescale (see Eq. 13 of Bolmont and Mathis 2016, note that $1 / T_{\star} \propto a^{-8}$ ). $K$ and $\omega_{\text {sat }}$ are parameters of the stellar wind model from Bouvier et al. (1997), which is valid for main sequence stars in the studied mass range. If $\Omega_{\star}>\omega_{\text {sat }}, \mu=1$ and if $\Omega_{\star}<\omega_{\text {sat }}, \mu=3$. Equation 2 becomes

$$
\frac{\mathrm{d} \Omega_{\star}}{\mathrm{d} t}=\frac{1}{I_{\star}}\left[-\dot{I}_{\star} \Omega_{\star}-K \Omega_{\star}^{\mu} \omega_{\mathrm{sat}}^{3-\mu}\left(\frac{R_{\star}}{R_{\odot}}\right)^{1 / 2}\left(\frac{M_{\star}}{M_{\odot}}\right)^{-1 / 2}+\frac{h}{2 T_{\star}}\left[1-\frac{\Omega_{\star}}{n}\right]\right] .
$$

If we write this equation in terms of the stellar rotation period $P_{\star}=2 \pi / \Omega_{\star}$, we get

$$
\begin{aligned}
\frac{\mathrm{d} P_{\star}}{\mathrm{d} t}= & \frac{-2 \pi}{I_{\star}}\left[-\dot{I}_{\star} \Omega_{\star}^{-1}-K \Omega_{\star}^{\mu-2} \omega_{\text {sat }}^{3-\mu}\left(\frac{R_{\star}}{R_{\odot}}\right)^{1 / 2}\left(\frac{M_{\star}}{M_{\odot}}\right)^{-1 / 2}\right. \\
& \left.+\frac{h}{2 T_{\star} \Omega_{\star}^{2}}\left[1-\frac{\Omega_{\star}}{n}\right]\right] .
\end{aligned}
$$

Let us consider an initially fast rotating Sun-like star $\left(M_{\star}=1 M_{\odot}, P_{\star, 0}=1.2\right.$ day). Figure 1 shows the orbital evolution of Jupiter mass planets around a Sun-like star and the evolution of the spin of the star. As in Bolmont and Mathis (2016), we consider that the initial time of our simulations is $5 \mathrm{Myr}$. The planets are distributed between an initial semimajor axis of $0.022 \mathrm{AU}$ and $0.04 \mathrm{AU}$. The planets, initially outside the corotation distance, migrate outwards and therefore by conservation of angular momentum act to slow down the contraction induced spin-up of the star. $\delta \Omega$ has a straightforward evolution; the star spins down when the planet is migrating outwards. The closer the planet is to the corotation distance, the farther the migration and the larger the spin-down of the star. Once the migration has stopped, the winds tend to spin down the star faster which rotates faster (the star without planet) and spin down the star slower which rotates slower (the star with planet). This leads to a convergence towards 0 of $\delta \Omega$.

However, the evolution of $\delta P$ is not as straightforward. It increases as it should until an age of $\sim 10 \mathrm{Myr}$, decreases until the end of the contraction phase (where the stellar rotation is the fastest), increases significantly until $\sim 500 \mathrm{Myr}$ (when the star passes from a saturated regime to an unsaturated one) and then decreases towards 0 .

To explain this behaviour, let us consider two stars 1 and 2 of same age but different rotation periods, $P_{1}=2 \pi / \Omega_{1}$ and $P_{2}=2 \pi / \Omega_{2}$. As the stars have the same age, they have the same radius $R_{1}=R_{2}=R_{\star}$ and moment of inertia $I_{1}=I_{2}=I_{\star}$. (Here, we neglect 


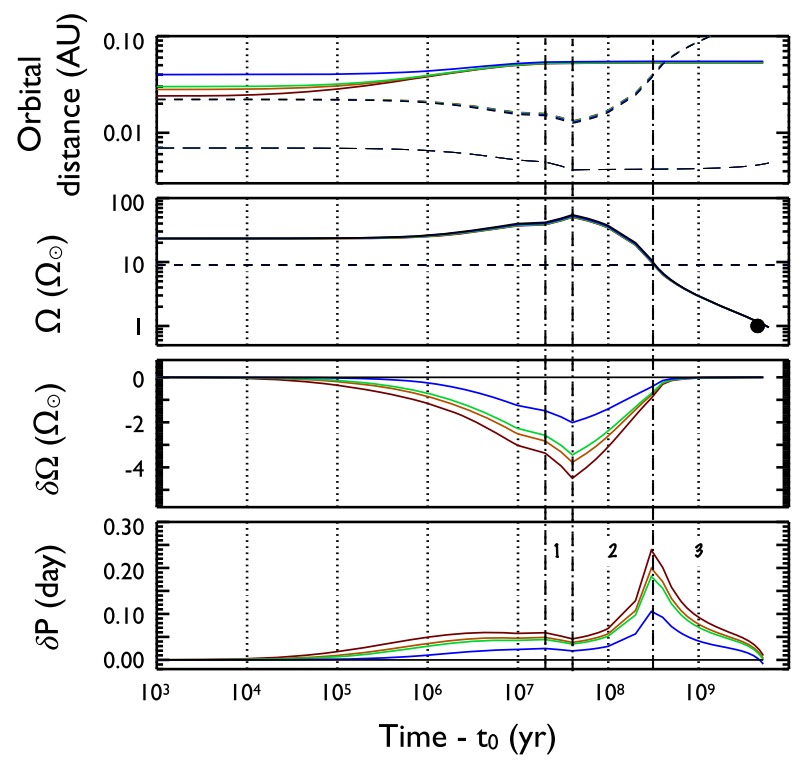

Fig. 1 Tidal evolution of Jupiter mass planets around an initially fast rotating Sun-like star. Top panel: evolution of the semimajor axis of the planet (coloured full lines), corotation distance (dashed lines) and stellar radius (long-dashed lines). Middle top panel: evolution of the spin of the star (full lines) and the saturation spin $\omega_{\text {sat }}$ (dashed line). The black lines correspond to the case with no planet, and the present day rotation of the Sun is represented by the black dot. Middle bottom panel: evolution of $\delta \Omega$. Bottom panel: evolution of $\delta P$. Note that $\delta \Omega$ for the closest planet (in blue) also changes sign when $\delta P$ does towards the end of the evolution; however, the scale allowing us to display the full evolution of $\delta \Omega$ does not allow to show this sign reversal

the impact of rotation on stellar structure and evolution; for example, Maeder (2009) and references therein.) From Eq. 4, we can compute the derivative of $\Delta P=P_{1}-P_{2}$

$$
\frac{\mathrm{d} \Delta P}{\mathrm{~d} t}=\frac{2 \pi}{I_{\star}} \frac{\Omega_{2}-\Omega_{1}}{\Omega_{1} \Omega_{2}} \times \begin{cases}\dot{I}_{\star}-K \Omega_{1} \Omega_{2}\left(\frac{R_{\star}}{R_{\odot}}\right)^{1 / 2}\left(\frac{M_{\star}}{M_{\odot}}\right)^{-1 / 2}, & \text { if }\left\{\Omega_{1}, \Omega_{2}\right\}<\omega_{\text {sat }}, \\ \dot{I}_{\star}+K \omega_{\text {sat }}^{2}\left(\frac{R_{\star}}{R_{\odot}}\right)^{1 / 2}\left(\frac{M_{\star}}{M_{\odot}}\right)^{-1 / 2}, & \text { if }\left\{\Omega_{1}, \Omega_{2}\right\}>\omega_{\text {sat }} .\end{cases}
$$

Let us consider that star 1 spins slower than star 2 (e.g. star 1 had an outward-migrating planet and star 2 has no planet) so that $\Omega_{1}<\Omega_{2}$. If the two stars are in the saturated regime, then $\frac{\mathrm{d} \Delta P}{\mathrm{~d} t}$ has the sign of $\dot{I}_{\star}+K \omega_{\text {sat }}^{2}\left(\frac{R_{\star}}{R_{\odot}}\right)^{1 / 2}\left(\frac{M_{\star}}{M_{\odot}}\right)^{-1 / 2}$. This means that there is a critical derivative of the moment of inertia $\dot{I}_{\text {crit }}$ defined as

$$
\dot{I}_{\text {crit }}=-K \omega_{\text {sat }}^{2}\left(\frac{R_{\star}}{R_{\odot}}\right)^{1 / 2}\left(\frac{M_{\star}}{M_{\odot}}\right)^{-1 / 2}
$$

If the two stars are in the unsaturated regime, then $\frac{\mathrm{d} \Delta P}{\mathrm{~d} t}$ has the sign of $\dot{I}_{\star}-$ $K \Omega_{1} \Omega_{2}\left(\frac{R_{\star}}{R_{\odot}}\right)^{1 / 2}\left(\frac{M_{\star}}{M_{\odot}}\right)^{-1 / 2}$. In our example, the unsaturated regime is reached when 


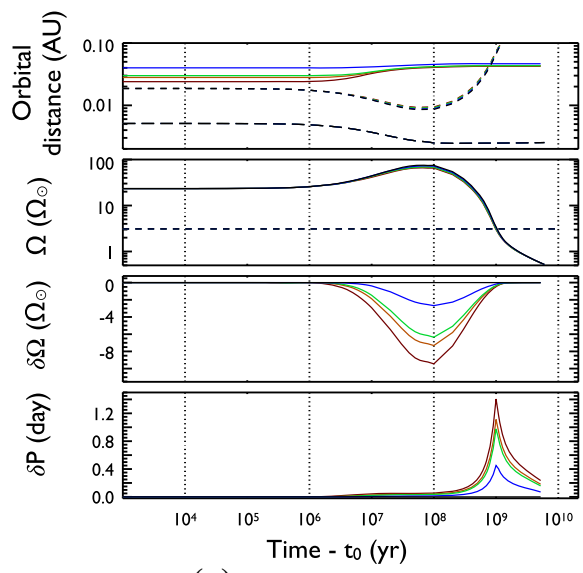

(a) $M_{\star}=0.6 M_{\odot}$

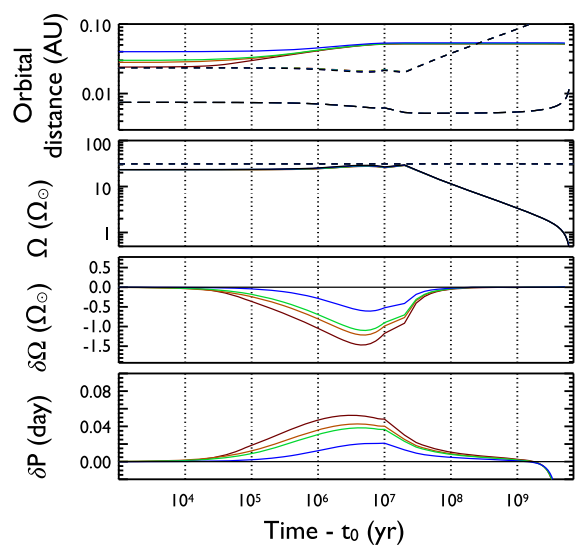

(b) $M_{\star}=1.2 M_{\odot}$

Fig. 2 Same as Fig. 1 but for different masses: a $0.6 M_{\odot}$, b $1.2 M_{\odot}$. Note that the scales are different for the two cases

the star is on the main sequence so when $\dot{I}_{\star} \approx 0$, so that here $\frac{\mathrm{d} \Delta P}{\mathrm{~d} t}$ is negative. We can therefore identify the different phases of the evolution of $\delta P=P_{1}-P_{2}$.

- If the stars are in the saturated regime and if $\dot{I}_{\star}<\dot{I}_{\text {crit }}$, which is verified if the star experiences a strong contraction (i.e. just before the main sequence or in absolute values: $\left.\left|\dot{I}_{\star}\right|>\left|\dot{I}_{\text {crit }}\right|\right), \delta P$ decreases (the rotations converge): This is the phase number 1 of Fig. 1.

- If the stars are in the saturated regime and if $\dot{I}_{\star}>\dot{I}_{\text {crit }}$, which is verified if the stellar moment of inertia does not evolve much (i.e. during most of the PMS and the MS), $\delta P$ increases (the rotations diverge). This is what happens from the end of the contraction phase ( $\sim 50 \mathrm{Myr})$ to the unsaturated/saturated transition $(\sim 500 \mathrm{Myr})$ : This is the phase number 2 of Fig. 1.

- If the stars are in the unsaturated regime and on the main sequence, $\delta P$ decreases (the rotations converge): This is the phase number 3 of Fig. 1.

- If the stars are in the unsaturated regime and at the beginning of the giant branch where the radius of the star increases sufficiently so that $\dot{I}_{\star}>K \Omega_{1} \Omega_{2}\left(\frac{R_{\star}}{R_{\odot}}\right)^{1 / 2}\left(\frac{M_{\star}}{M_{\odot}}\right)^{-1 / 2}$, $\delta P$ increases (the rotations diverge). We can see this effect starting to operate as the slope of $\delta P$ changes its inflection around $3 \mathrm{Gyr}$ (bottom panel of Fig. 1). But this trend cannot really be seen because at late ages, the planets begin to fall towards the star making it accelerate slightly.

Figure 2 shows the evolution of Jupiter mass planets around stars of different masses, $M_{\star}=0.6 M_{\odot}$ and $M_{\star}=1.2 M_{\odot}$. As expected, the effect of the planet on the stellar rotation decreases as stellar mass increases. For $M_{\star}=0.6 M_{\odot}$, the evolution is similar to the evolution for $1 M_{\odot}$, with a maximum of $\delta P$ occurring at the transition saturated/unsaturated. For $M_{\star}=1.2 M_{\odot}$, the star is always in the unsaturated regime so the shape is less complex. The wind is here acting to damp the effect of the planet even before the migration has finished.

As explained in Bolmont and Mathis (2016), due to the different values of the stellar dissipation and its evolution, the migration occurs faster for the more massive star and on longer timescales for the less massive star. This means that the maximum of $\delta P$ occurs earlier for the more massive star and later for the less massive star. 

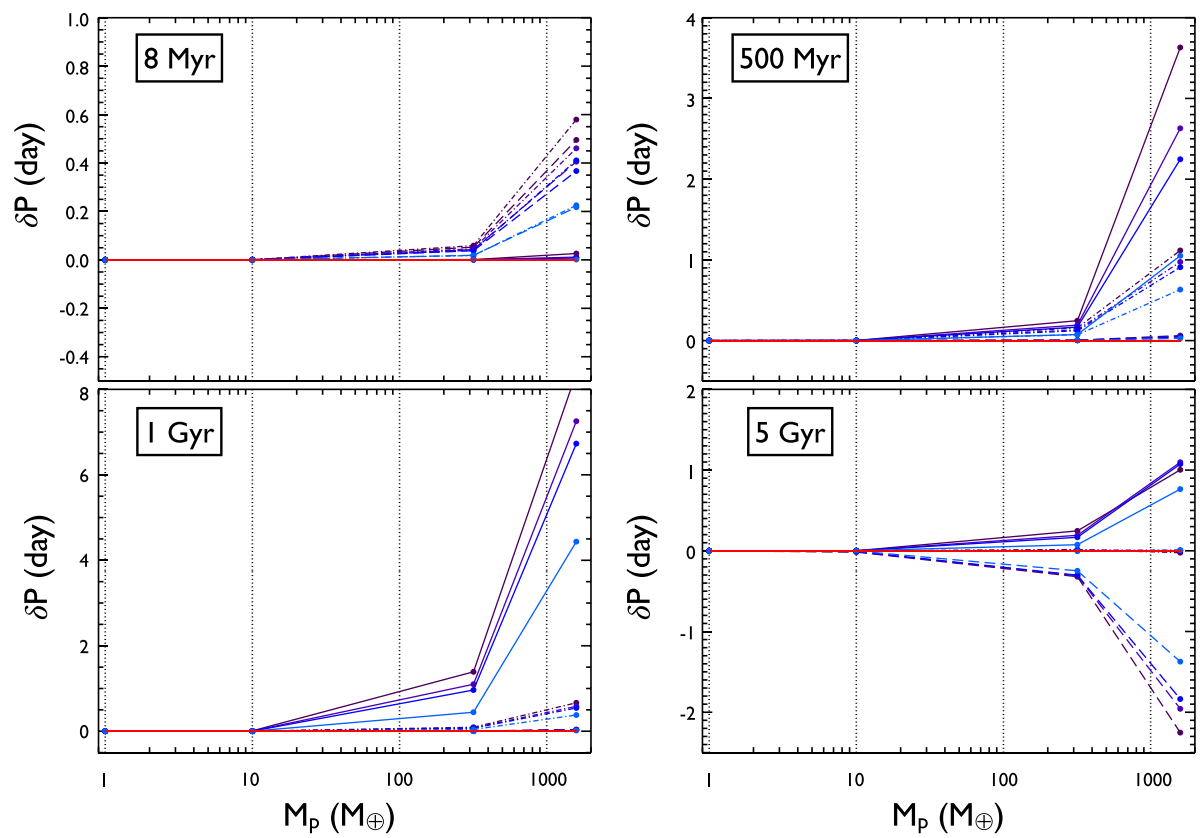

Fig. $3 \delta P$ for different initial orbital distances and for the 3 different stars and 4 different ages. The solid lines correspond to the $0.6 M_{\odot}$ star, the dashed-dotted lines correspond to $1.0 M_{\odot}$ and the long-dashed lines correspond to $1.2 M_{\odot}$. From purple to light blue: an initial semimajor axis of 0.024, 0.028, 0.030 and 0.040 AU. The red line marks $\delta P=0$ day. Note that the scales are different for each panel

In order to represent the evolution of $\delta P$ with the age of the star, we updated in Fig. 3 the Fig. 5 of Bolmont and Mathis (2016) for four different ages: at $8 \mathrm{Myr}$ (around the maximum effect for the star of $1.2 M_{\odot}$ ), at $500 \mathrm{Myr}$ (around the maximum effect for the star of $1.0 M_{\odot}$ ), at $1 \mathrm{Gyr}$ (around the maximum effect for the star of $0.6 M_{\odot}$ ) and at $5 \mathrm{Gyr}$ (the age used for Fig. 5 of Bolmont and Mathis 2016). We consider planet masses from the mass of Earth to five times the mass of Jupiter.

The effect of the planet on the star is much less pronounced than what was shown in Fig. 5 of Bolmont and Mathis (2016). The effect of a Jupiter mass planet on the rotation of a $0.6 M_{\odot}$ star is of 0.1 to 0.3 days at $5 \mathrm{Gyr}$, while Bolmont and Mathis (2016) was showing an effect of 2.2 to 3 days. Note also that for the $1.2 M_{\odot}$ star, the stars with planets rotate faster than the star without planets. This coincides with the beginning of the red giant branch when the radius of the star starts to increase (see Fig. 2b) and the planets have already started to migrate inwards.

However, the planet can still have quite an important impact on the rotation towards the end of the outward migration. This is thus an effect which highly depends on the age of the star and which could eventually be observable via high-precision photometry (McQuillan et al. 2013; García et al. 2014; Ceillier et al. 2016).

Acknowledgements The authors would like to thank the anonymous referee for his comments on this manuscript. E. B. and S. M. acknowledge funding by the European Research Council through ERC Grant SPIRE 647383. 


\section{References}

Bolmont, E., Mathis, S.: Celest. Mech. Dyn. Astr. 126, 275 (2016)

Bouvier, J., Forestini, M., Allain, S.: A\&A 326, 1023 (1997)

Ceillier, T., van Saders, J., García, R.A., et al.: MNRAS 456, 119 (2016)

García, R.A., Ceillier, T., Salabert, D., et al.: A\&A 572, A34 (2014)

Maeder, A.: Physics. Formation and Evolution of Rotating Stars, Springer, Berlin Heidelberg (2009)

McQuillan, A., Mazeh, T., Aigrain, S.: ApJ 775, L11 (2013)

Siess, L., Dufour, E., Forestini, M.: A\&A 358, 593 (2000)

Publisher's Note Springer Nature remains neutral with regard to jurisdictional claims in published maps and institutional affiliations. 